R. Pant, C. G. Poulton, H. MacFarlane, L. Thevenaz, D. Choi, S. J. Madden, B. Luther-Davies, and B. Eggleton, "On-chip stimulated Brillouin scattering," in CLEO:2011 - Laser Applications to Photonic Applications, OSA Technical Digest (CD) (Optical Society of America, 2011), paper CTuX5. 


\title{
On-chip stimulated Brillouin scattering
}

\author{
${ }^{1,4}$ Ravi Pant, ${ }^{2,4}$ Christopher Poulton, ${ }^{1,4}$ Hannah MacFarlane, ${ }^{5}$ Luc Thevenaz, ${ }^{3,4}$ Duk-Yong Choi, \\ ${ }^{3,4}$ Steve J. Madden, ${ }^{3,4}$ Barry Luther-Davies, and ${ }^{1,4}$ Benjamin J. Eggleton \\ ${ }^{I}$ School of Physics, University of Sydney, Sydne, NSW 2006, Australia \\ ${ }^{2}$ University of Technology Sydney, Sydney, NSW 2007, Australia \\ ${ }^{3}$ Laser Physics Centre, Australian National University, Canberra, ACT 0200, Australia \\ ${ }^{4}$ Centre for Ultrahigh bandwidth Devices for Optical Systems, ARC Centre of Excellence, Australia \\ ${ }^{5}$ Institute of Electrical Engineering, Ecole Polytechnique Fédérale de Lausanne, 1015 Lausanne, Switzerland \\ email:rpant@physics.usyd.edu.au
}

\begin{abstract}
We report the first demonstration of on-chip stimulated Brillouin scattering (SBS). The measured Brillouin shift and line width are $\sim 7.7 \mathrm{GHz}$ and $6 \mathrm{MHz}$ in a $7 \mathrm{~cm}$ long chalcogenide waveguide.

(C)2010 Optical Society of America

OCIS codes: $190.0190,190.4360,190.5890$
\end{abstract}

Stimulated Brillouin scattering (SBS) is a nonlinear process resulting from the interaction between light and acoustic modes in an optically transparent structure [1]. SBS has been exploited in a number of applications ranging from slow-light to Brillouin lasers using long length $(\sim \mathrm{km})$ optical fibers [2, 3]. However, the investigation of on-chip SBS, in which the effect is induced in an integrated optical structure, has been limited because most platforms commonly used in nonlinear optics either have very small SBS gain coefficient (as is the case for Silica) or have no SBS even though they have very high Kerr nonlinearity (e.g. for Silicon). Chalcogenide glass, on the other hand, has both large SBS gain coefficient and Kerr nonlinearity [4]. SBS has been measured in chalcogenide fibers and the measured Brillouin shift and linewidth are $7.985 \mathrm{GHz}$ and $13 \mathrm{MHz}$ respectively [4]. We present the first demonstration of on-chip SBS in a $7 \mathrm{~cm}$ long, silica-clad chalcogenide $\left(\mathrm{As}_{2} \mathrm{~S}_{3}\right)$ rib waveguide. The measured Brillouin shift and linewidth are $\sim 7.72 \mathrm{GHz}$ and $\sim 6 \mathrm{MHz}$ respectively, consistent with previous results in chalcogenide fibers.

Figure 1 shows a schematic of on-chip SBS process where a pump beam at frequency $\left(\omega_{\mathrm{p}}\right)$ is launched into the $\mathrm{As}_{2} \mathrm{~S}_{3}$ optical-chip and is backscattered by the acoustic wave of frequency $\left(\Omega_{\mathrm{B}}\right)$. The scattered light, known as the Stokes wave, appears at a frequency $\omega_{\mathrm{s}}=\omega_{\mathrm{p}}-\Omega_{\mathrm{B}}$. Figure 1 shows the cross-section of the $\mathrm{As}_{2} \mathrm{~S}_{3}$ waveguide and the optical and acoustic modes of the waveguide obtained using the finite element method.

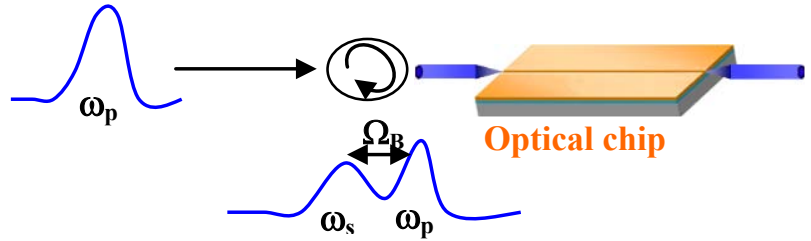

Figure1. Principal of on-chip stimulated Brillouin scattering

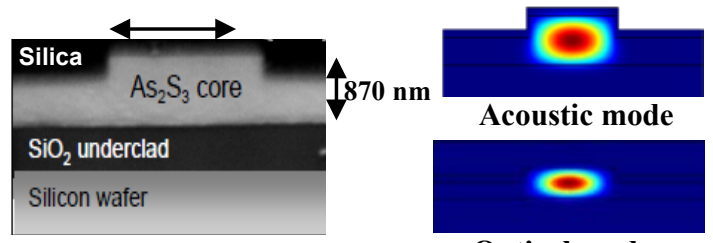

Optical mode

The large Brillouin gain coefficient $\left(\mathrm{g}_{\mathrm{B}}\right)$ for $\mathrm{As}_{2} \mathrm{~S}_{3}$ has its origin in its large refractive index $(\mathrm{n}=2.44)$. The gain coefficient $\mathrm{g}_{\mathrm{B}}$ depends on $n$ according to $[1,4]$

$$
g_{B}=I 2 \pi n^{7} P^{2}{ }_{12} /\left(c \rho v_{a} \Delta v_{B} \lambda_{p}^{2}\right),
$$

where $I$ is the overlap integral between the acoustic and optical mode, $P_{12}$ is the longitudinal elasto-optic coefficient, $\mathrm{c}$ is the speed of light in vacuum, $\rho$ is the material density, $v_{a}$ is the acoustic speed, $\Delta v_{\mathrm{B}}$ is the Brillouin linewidth. The value of $g_{B}$ is obtained by solving the acoustic and optical modes for the waveguide and calculating the overlap integral and has a value of $\mathrm{g}_{\mathrm{B}} \sim 2 \times 10^{-9} \mathrm{~m} / \mathrm{W}$.

Figure 2 shows the experimental set-up for investigating SBS in $\mathrm{As}_{2} \mathrm{~S}_{3}$ chip. Light from a DFB laser at a pump wavelength $\left(\lambda_{\mathrm{p}}\right)$ of $1550.025 \mathrm{~nm}$ is modulated, after propagating it through a fiber polarization controller (FPC), using a $25 \mathrm{kHz}$ pulse train with a duty cycle of $1 \%$ to generate $400 \mathrm{~ns}$ pump pulses. Pump pulses are amplified using an EDFA and coupled to the circulator. A lensed fiber at port 2 of the circulator is used to couple light to the waveguide. Back scattered light is collected at port 3 of the circulator and sent to an RF spectrum analyzer and an 


\section{CTuX5.pdf}

optical spectrum analyzer (OSA) using a 90/10 splitter. The waveguide is $7 \mathrm{~cm}$ long and has a cross-sectional area of $4 \mu \mathrm{m} \times 870 \mathrm{~nm}$ with a top silica cladding of $140 \mathrm{~nm}$.

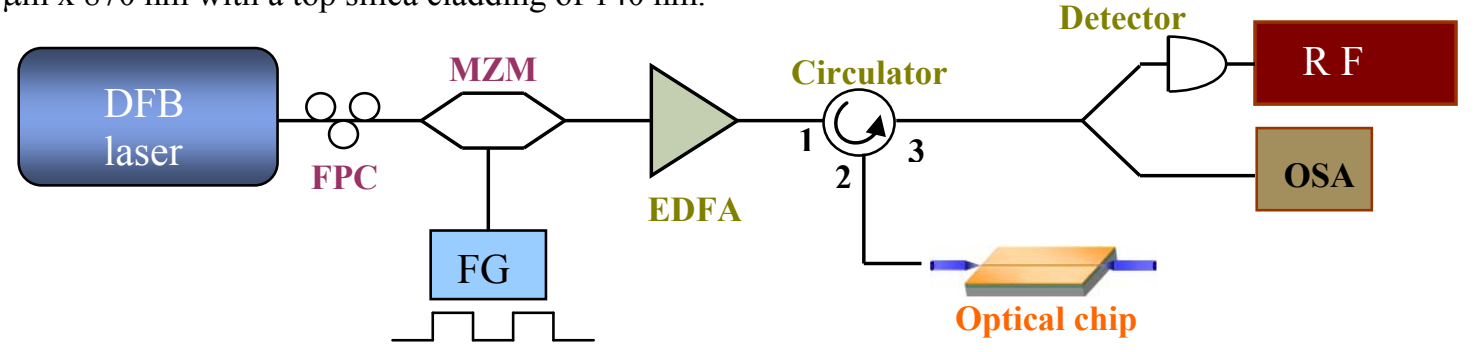

Figure 2 Experimental set-up for investigating on-chip SBS.

Figure 3 shows the back-scattered optical spectra for the $\mathrm{As}_{2} \mathrm{~S}_{3}$ waveguides for different input average powers before coupling to the waveguide. From Fig. 3 it is evident that the Stokes signal starts to appear as the pump power is increased. Figure 4 shows the RF spectra corresponding to the minimum and maximum input powers. From Fig. 4, we note that both the Brillouin shift and line width are slightly different for these two powers. The Brillouin shift for the minimum and maximum input powers, as inferred from the RF spectra, are $7.72 \mathrm{GHz}$ and $7.73 \mathrm{GHz}$ respectively and the corresponding $3 \mathrm{~dB}$ linewidths of the RF spectra are $\sim 6$ and $\sim 8 \mathrm{MHz}$ respectively. We attribute the broad line width and different shift at larger power to chip heating due to higher power dissipation in the waveguide. The measured Brillouin shift and linewidth are consistent with the measured values in chalcogenide fiber by Abedin $e t$ al. [4].

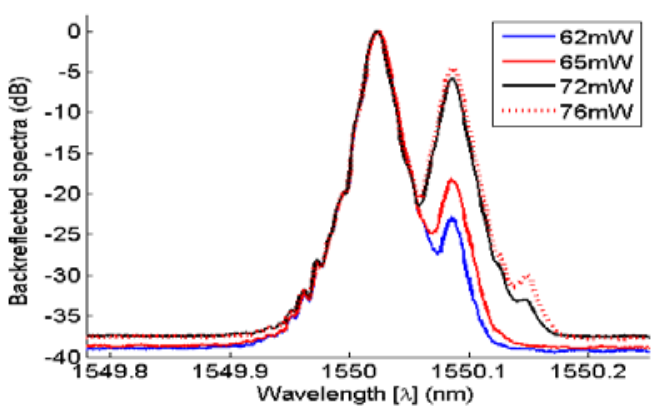

Figure 3 Backreflected spectra at different input powers for a 7 $\mathrm{cm}$ long $\mathrm{As}_{2} \mathrm{~S}_{3}$ waveguide showing the increase of the Stokes signal with power.

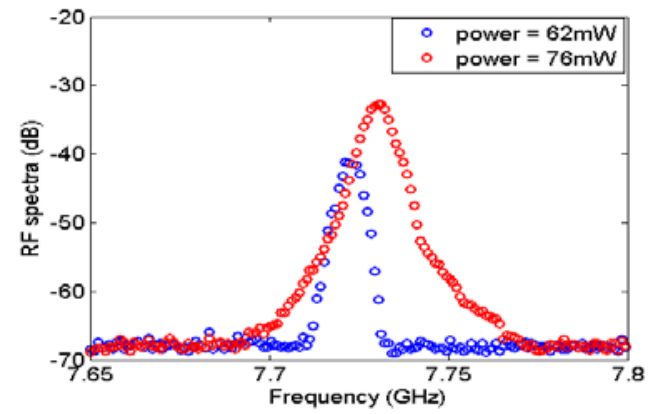

Figure 4 RF spectra at the minimum and maximum input power for a $7 \mathrm{~cm}$ long $\mathrm{As}_{2} \mathrm{~S}_{3}$ waveguide.

Finally, we calculate the Brillouin threshold for our $\mathrm{As}_{2} \mathrm{~S}_{3}$ waveguide using the expression [4]

$$
\mathrm{Kg}_{\mathrm{B}}\left(\mathrm{P}_{\mathrm{th}} / \mathrm{A}_{\text {eff }}\right) \mathrm{L}_{\text {eff }} \approx 21 \text {, }
$$

where $P_{t h}$ is the threshold pump power, $A_{\text {eff }}$ is the effective mode area, $K$ is a factor for taking into account the polarization effects and $L_{\text {eff }}$ is $(1-\exp (-\alpha \mathrm{L})) / \alpha$ with $\alpha$ and $L$ being the propagation loss and device length respectively. Table 1 compares the Brillouin threshold for our optical chip and the same length of single-mode fiber (SMF) using $\mathrm{K}=1$. Note that the Brillouin threshold for the optical chip is three orders of magnitude smaller than that for the same length of SMF owing to its 100 times larger $g_{B}$ and smaller mode area.

Table 1 Comparison of the Brillouin threshold for optical chip and same effective length of SMF.

\begin{tabular}{|l|l|l|l|l|}
\hline Device & $\mathrm{g}_{\mathrm{B}}(\mathrm{m} / \mathrm{W})$ & $\mathrm{L}_{\text {eff }}(\mathrm{cm})$ & $\mathrm{A}_{\text {eff }}\left(\mathrm{m}^{2}\right)$ & $\mathrm{P}_{\text {th }}(\mathrm{W})$ \\
\hline SMF & $2.0 \times 10^{-11}$ & 3 & $80 \times 10^{-12}$ & 2800 \\
\hline Optical chip & $2.0 \times 10^{-9}$ & 3 & $3 \times 10^{-12}$ & 1.0 \\
\hline
\end{tabular}

In conclusion, we have presented the first demonstration of on-chip SBS with low Brillouin threshold. On-chip SBS will enable chip-based devices for applications such as slow-light, signal processing.

References:

1. $\quad$ Robert W. Boyd, Nonlinear Optics. San Diego, CA: Academic press 2001.

2. Thevenaz et al., Nature Photonics, 2(8), 474-481 (2008).

3. Stokes et al., Optics Letters 7, 509-511 (1982).

4. Abedin et al. Optics Express 13, 10266-10271 (2005). 